Table of Contents

\title{
The First Total Synthesis of $\mathrm{J}_{2}$ Isoprostane
}

Giuseppe Zanoni*, Francesca Castronovo, Alessio Porta and Giovanni Vidari*

Dipartimento di Chimica Organica, Università di Pavia, Via Taramelli 10, 27100 Pavia, Italy

${ }^{1}$ H-NMR of compound 9: S2

${ }^{13} \mathrm{C}-\mathrm{NMR}$ of compound 9: S3

DEPT of compound 9: S4

${ }^{1} \mathrm{H}-\mathrm{NMR}$ of compound 16: S5

${ }^{13} \mathrm{C}-\mathrm{NMR}$ of compound 16: S6

DEPT of compound 16: S7

${ }^{1} \mathrm{H}-\mathrm{NMR}$ of compound 15: S8

${ }^{13} \mathrm{C}-\mathrm{NMR}$ of compound 15: S9

DEPT of compound 15: S10

${ }^{1}$ H-NMR of compound 14: S11

${ }^{13}$ C-NMR of compound 14: S12

DEPT of compound 14: S13

${ }^{1} \mathrm{H}-\mathrm{NMR}$ of compound 13: S14

${ }^{13} \mathrm{C}-\mathrm{NMR}$ of compound 13: S15

DEPT of compound 13: S16

${ }^{1}$ H-NMR of compound 1: S17

${ }^{13} \mathrm{C}-\mathrm{NMR}$ of compound $\mathbf{1}$ : S18 


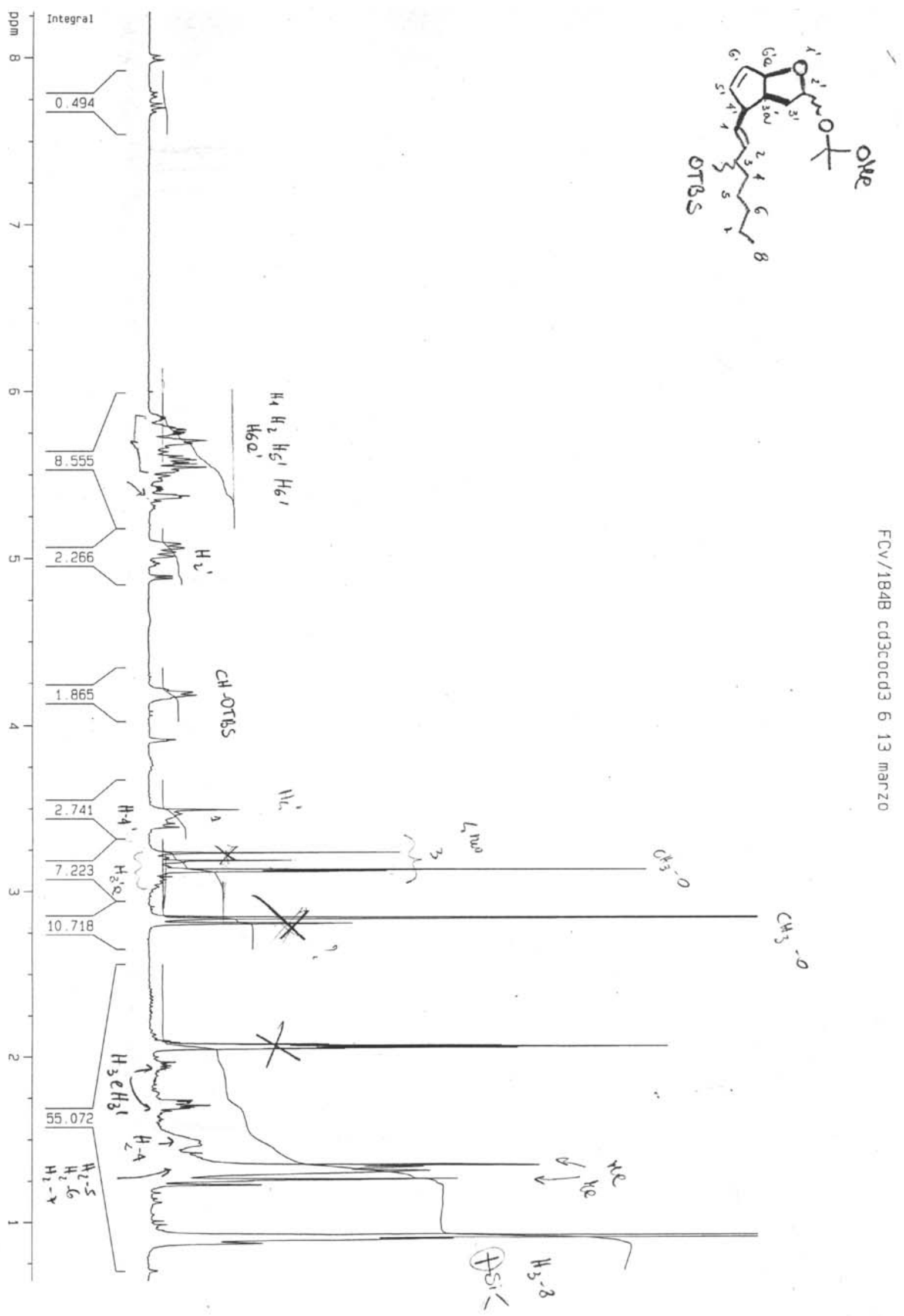




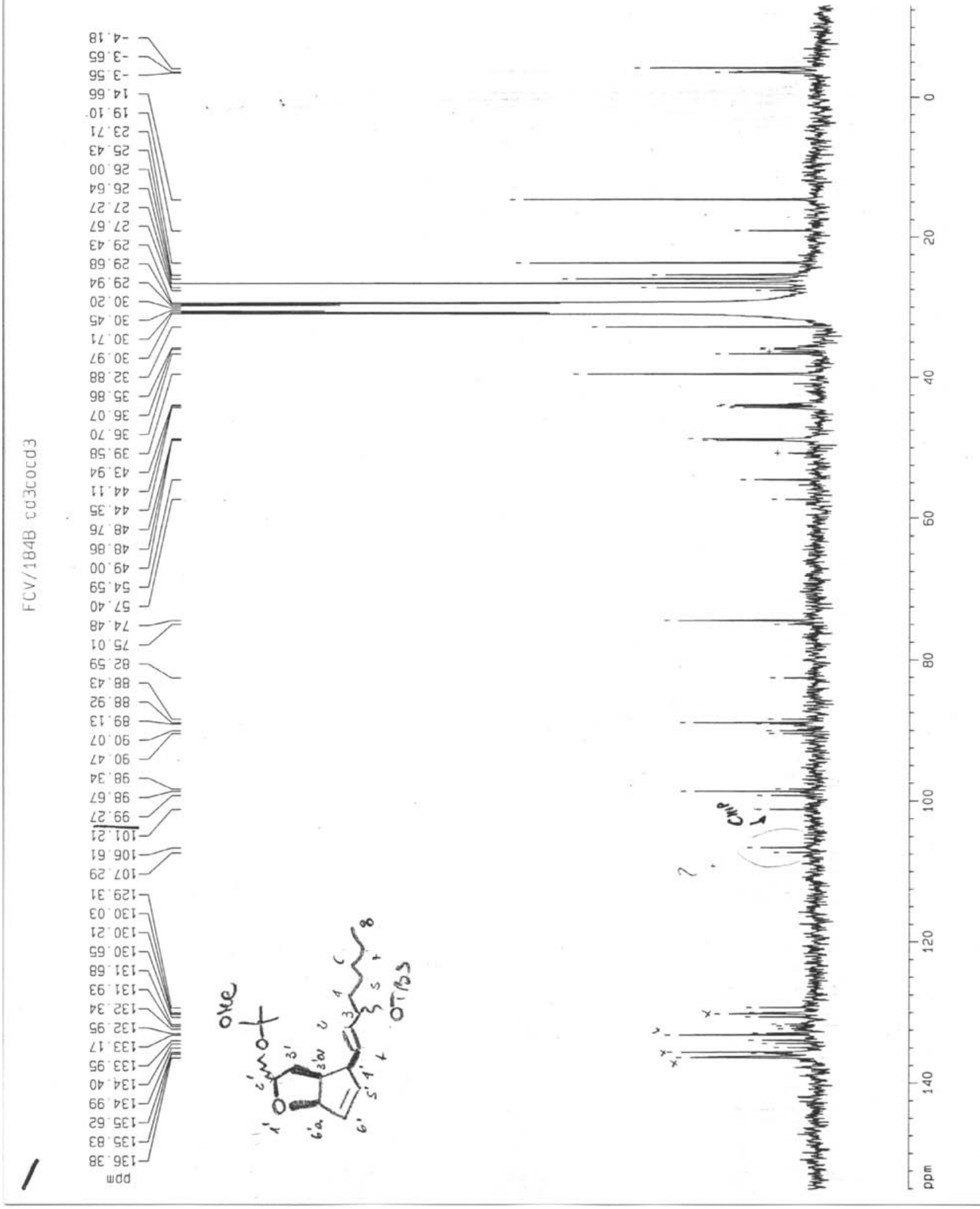




$$
1
$$




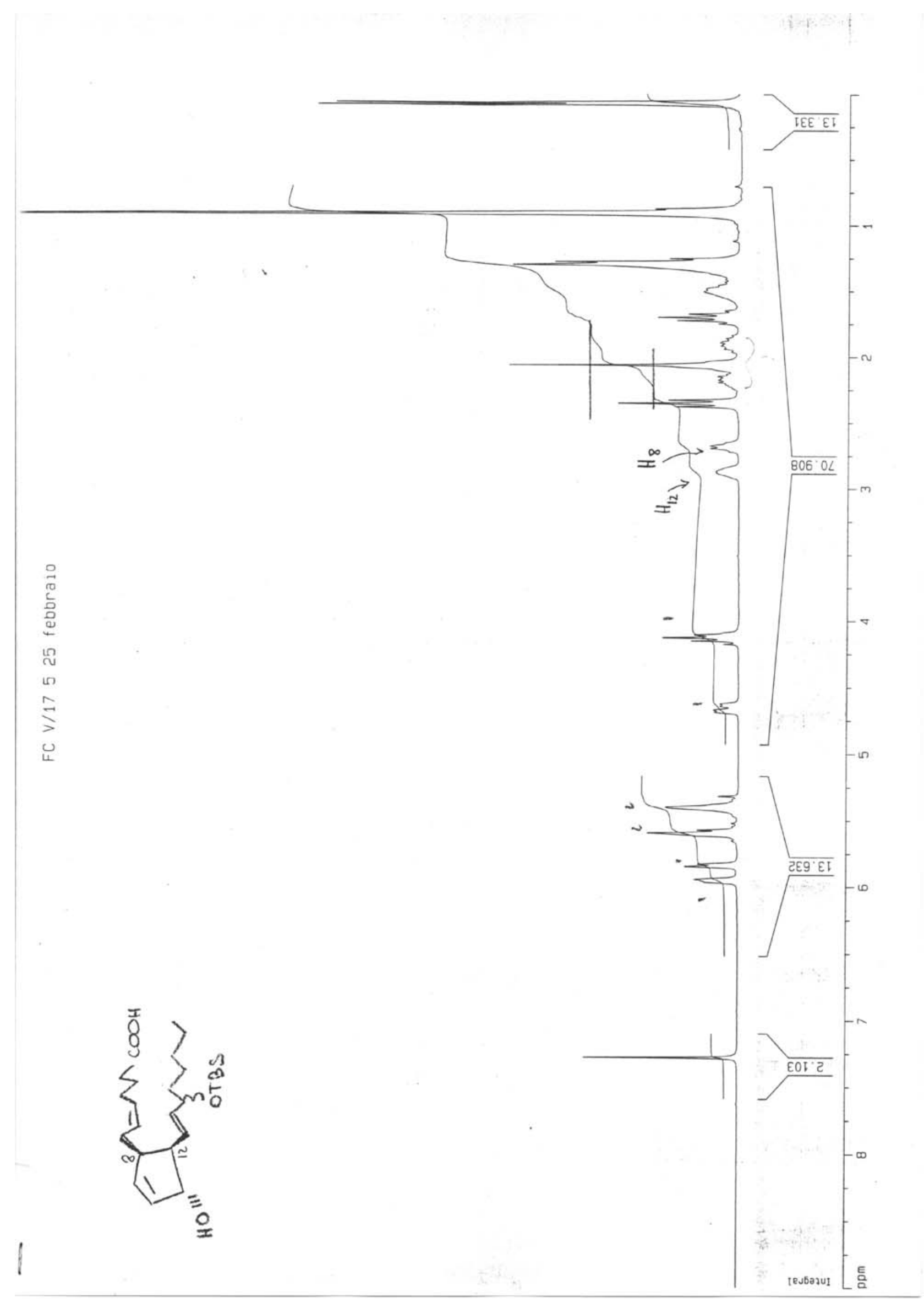




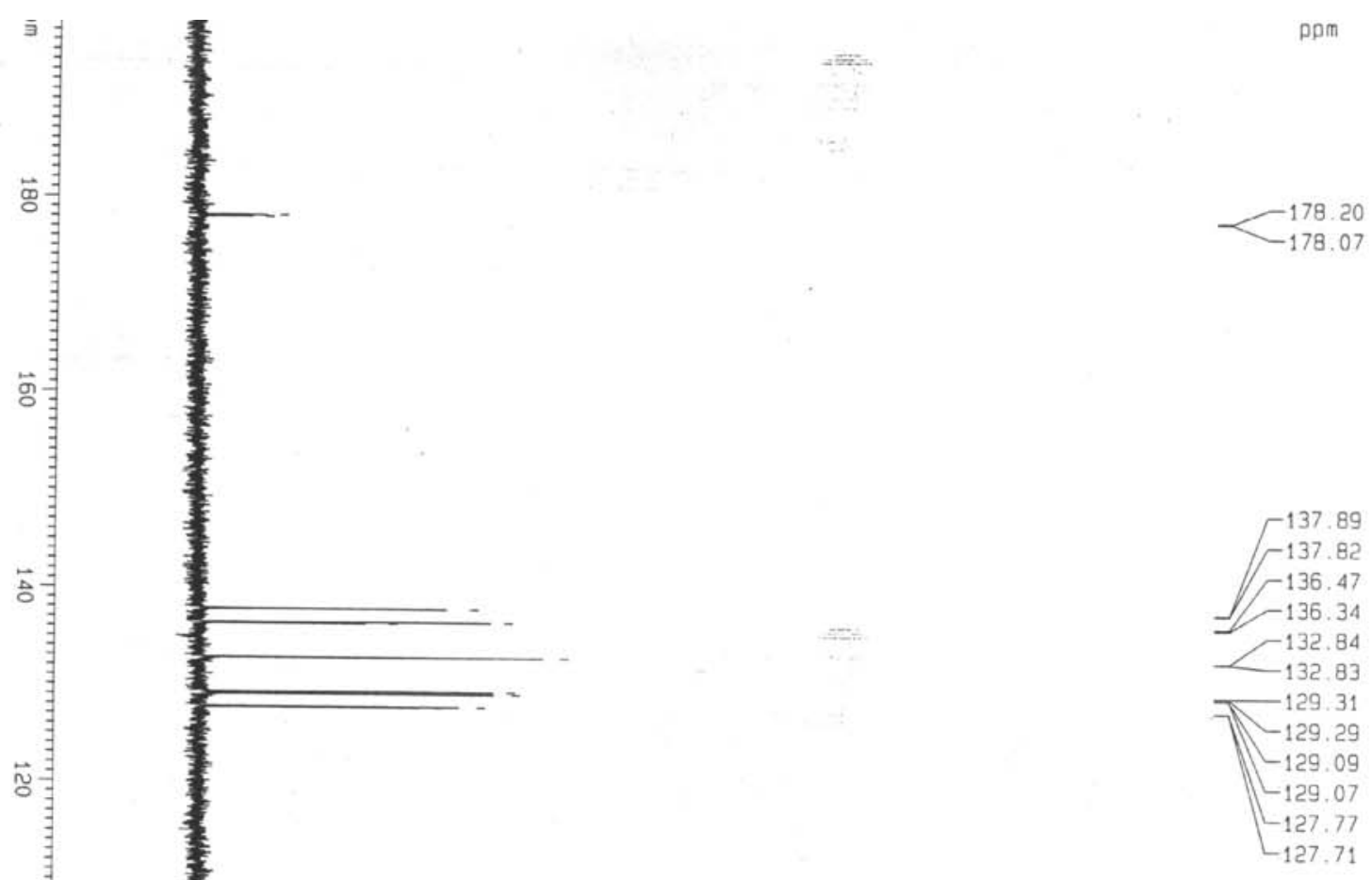

ஃ

回寻

$-81.63$

- 77.34

F 77.11

F 76.91

76.49

F 73.38

- 73.36

- 60.31

$-54.98$

- 54.91

- 47.98

$\left[\begin{array}{r}47.98 \\ -37.91\end{array}\right.$

- 38.30

- 38.22

- 33.06

] 33.02

If 31.73

- 31.70

$\Gamma^{29.06}$

- 28.98

${ }^{26.46}$

- 26.44

25.81
-24.98

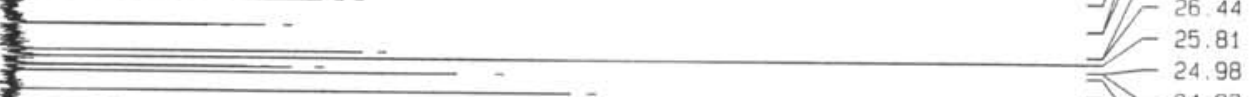

กำ

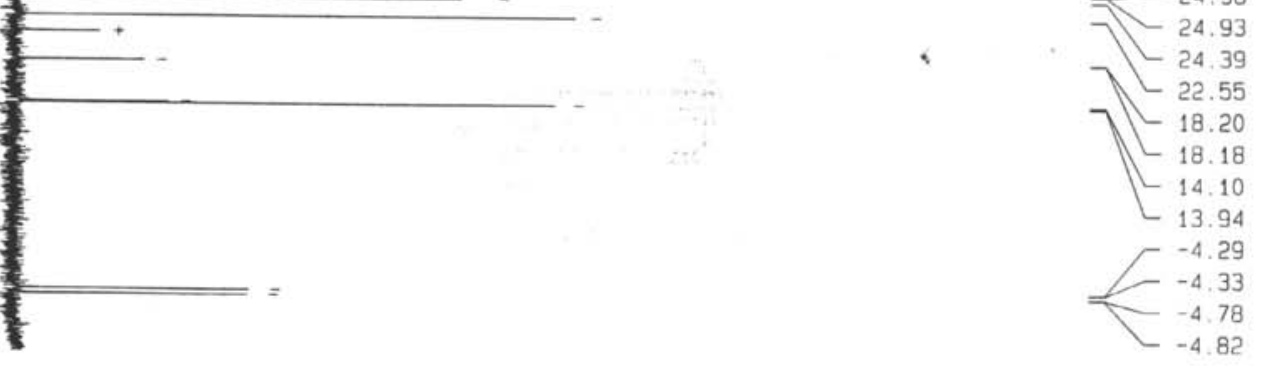




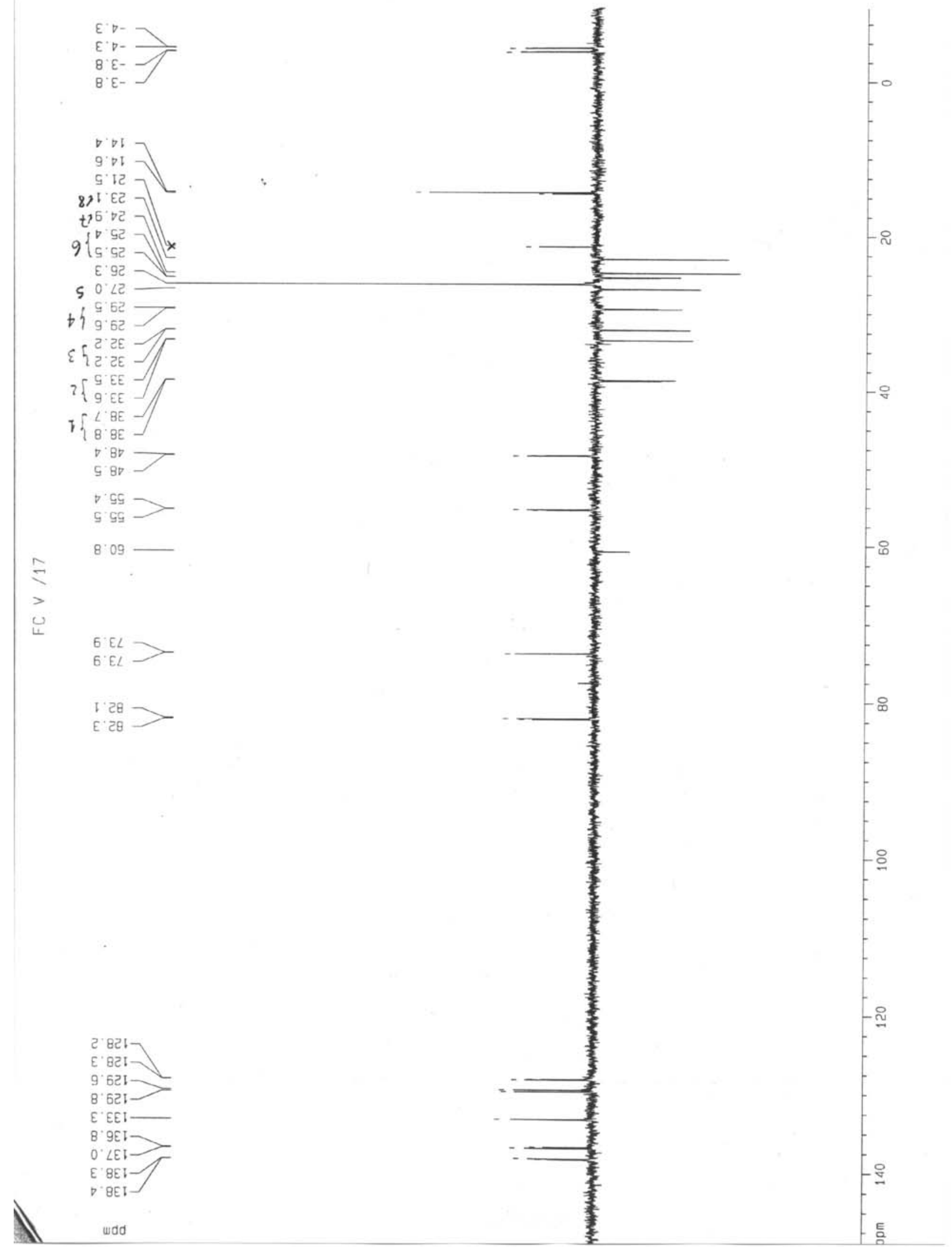




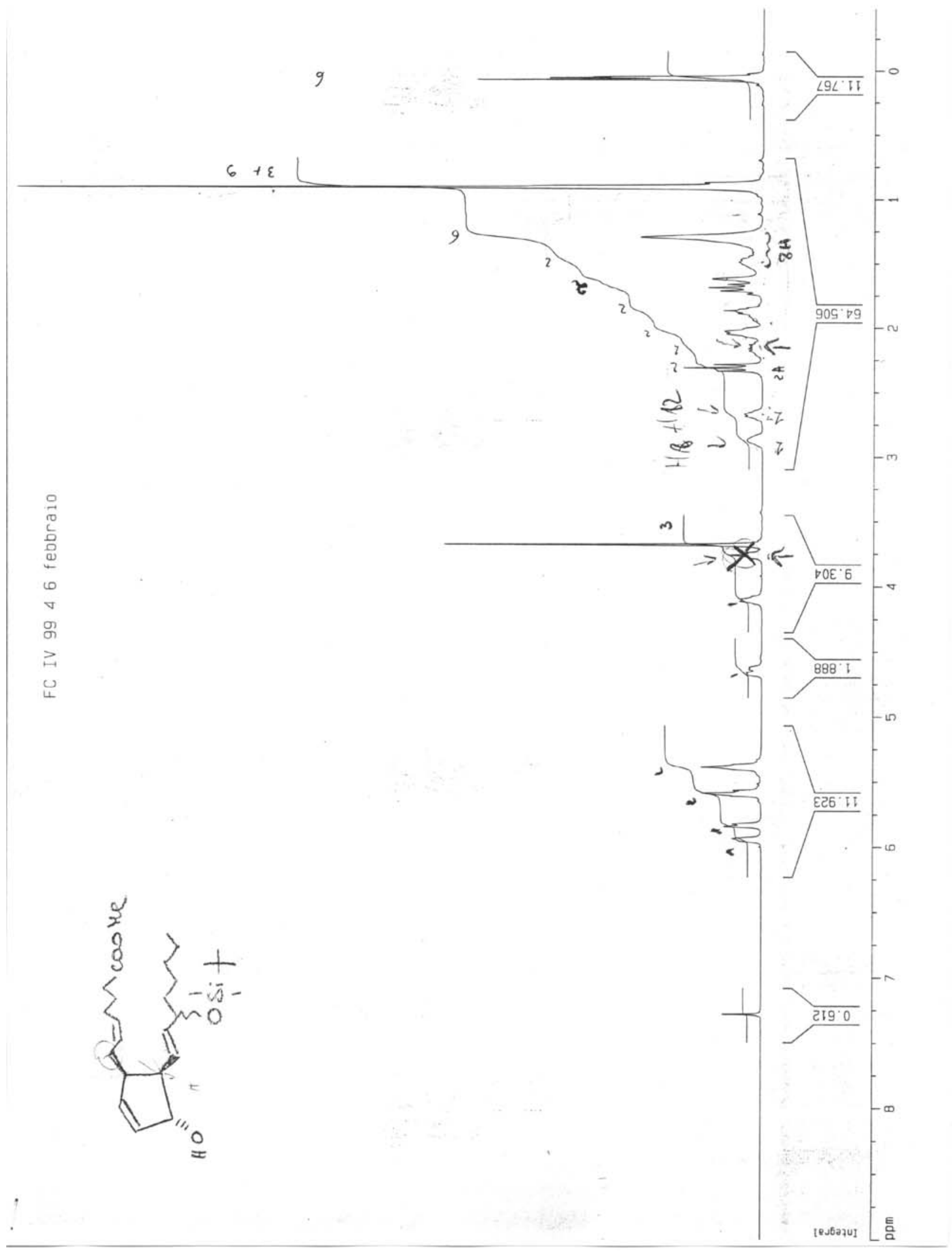





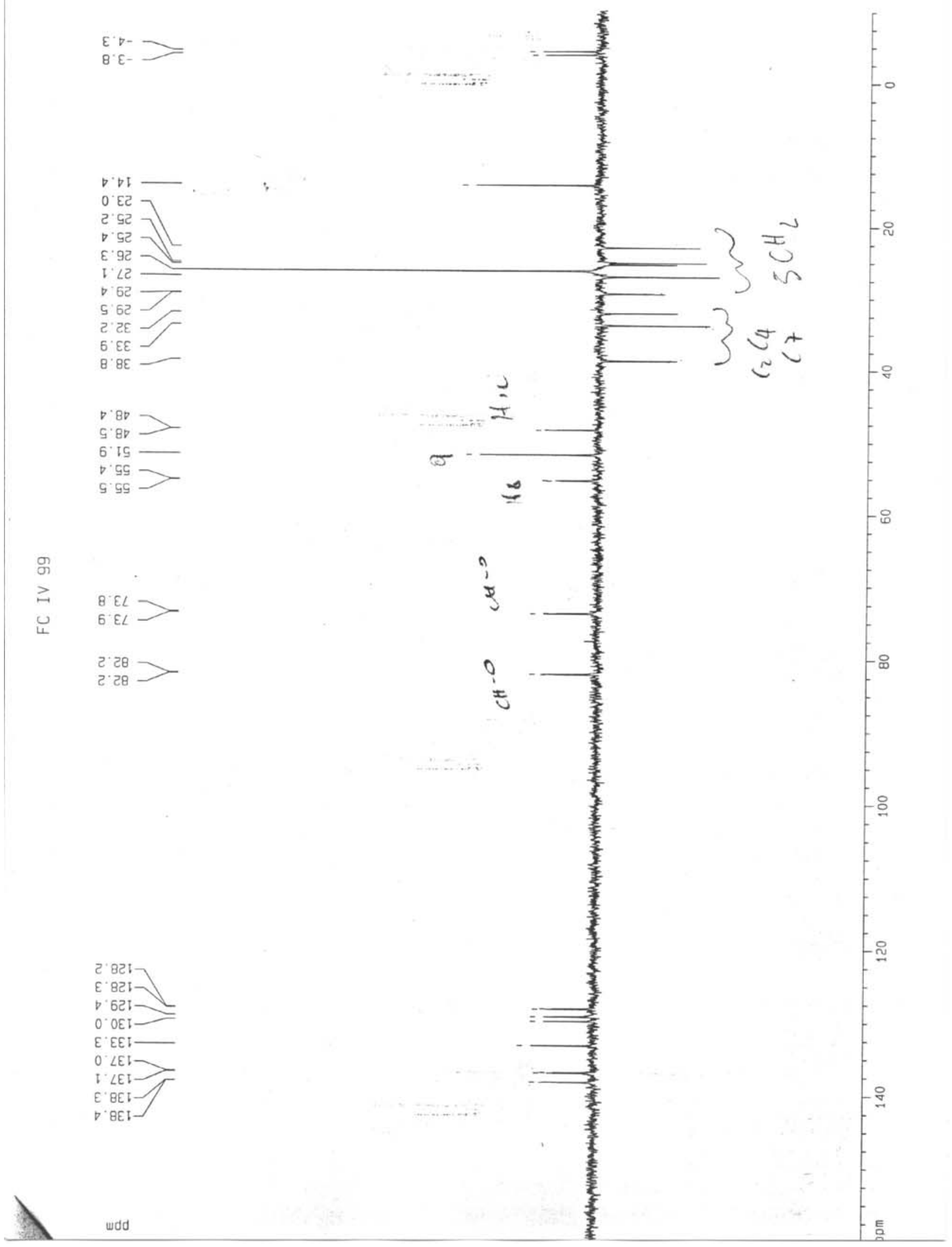




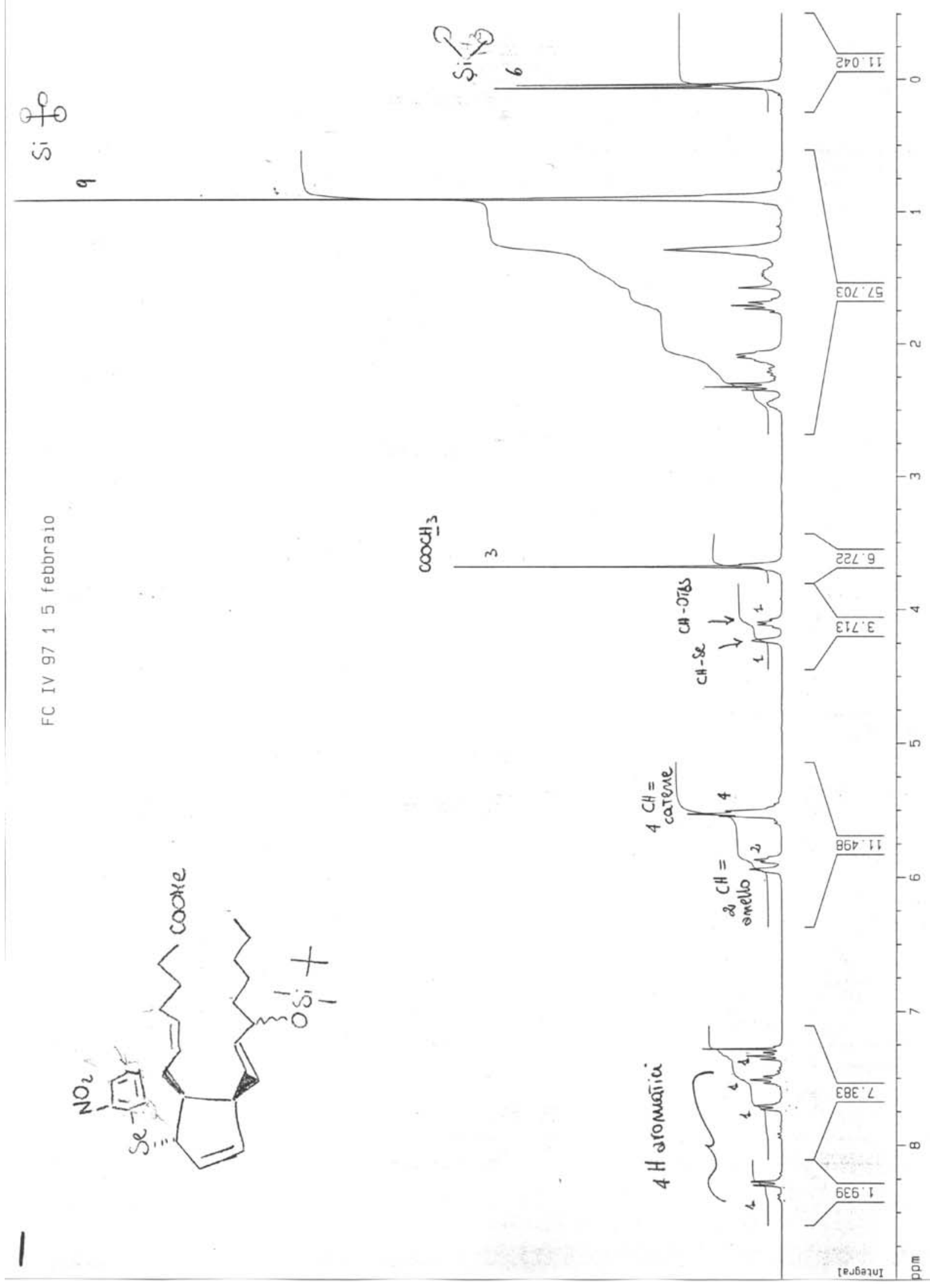




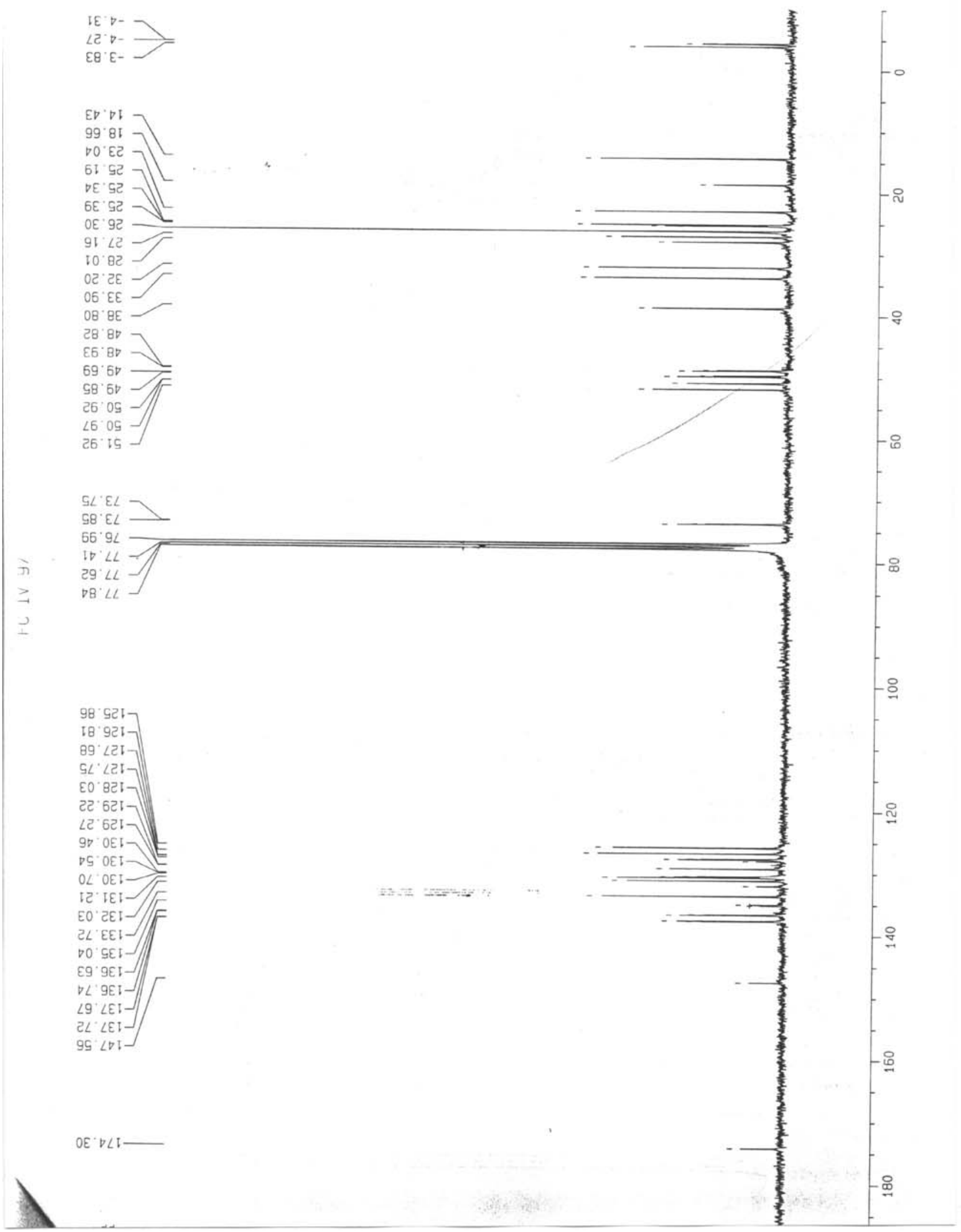




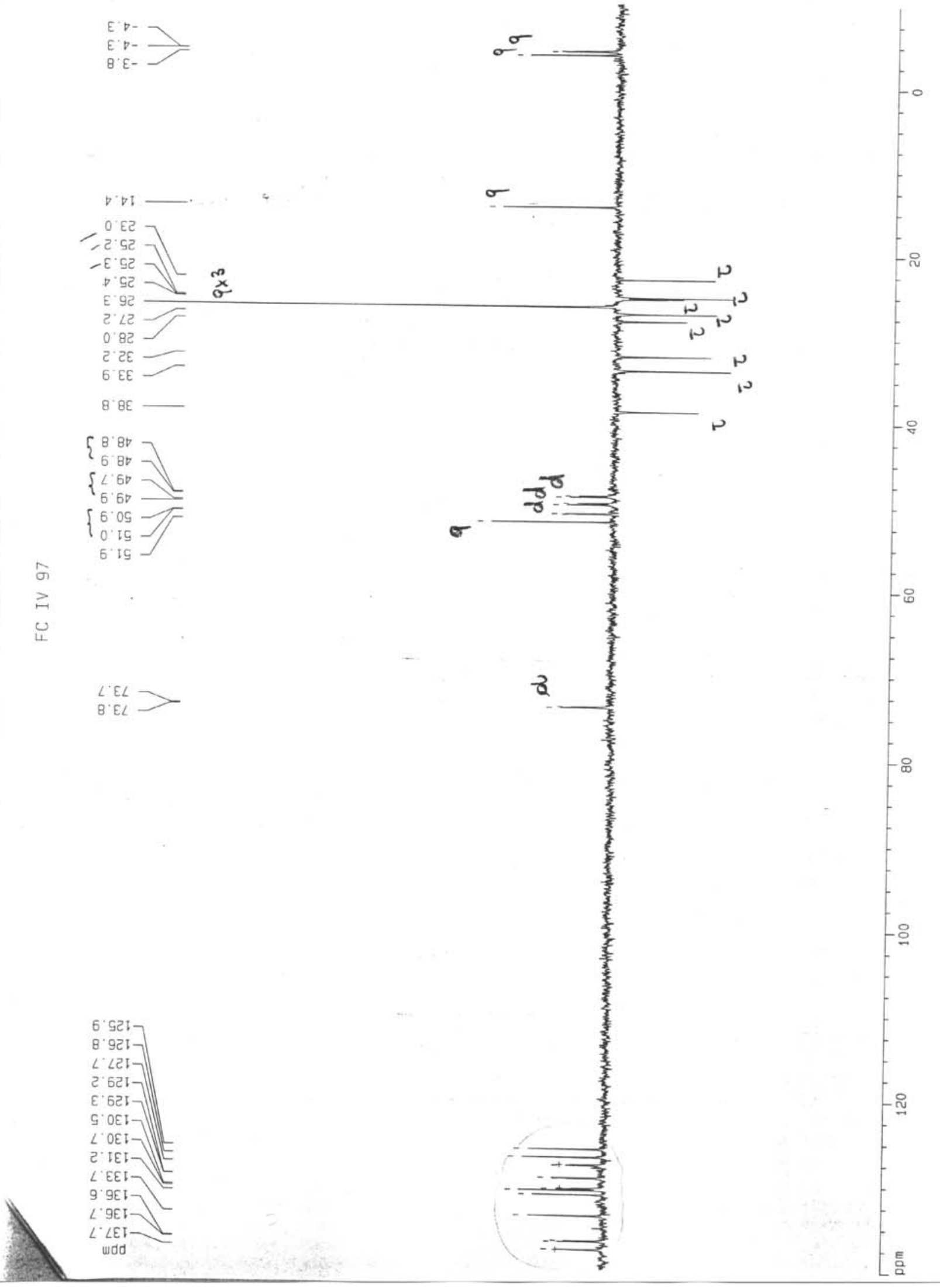




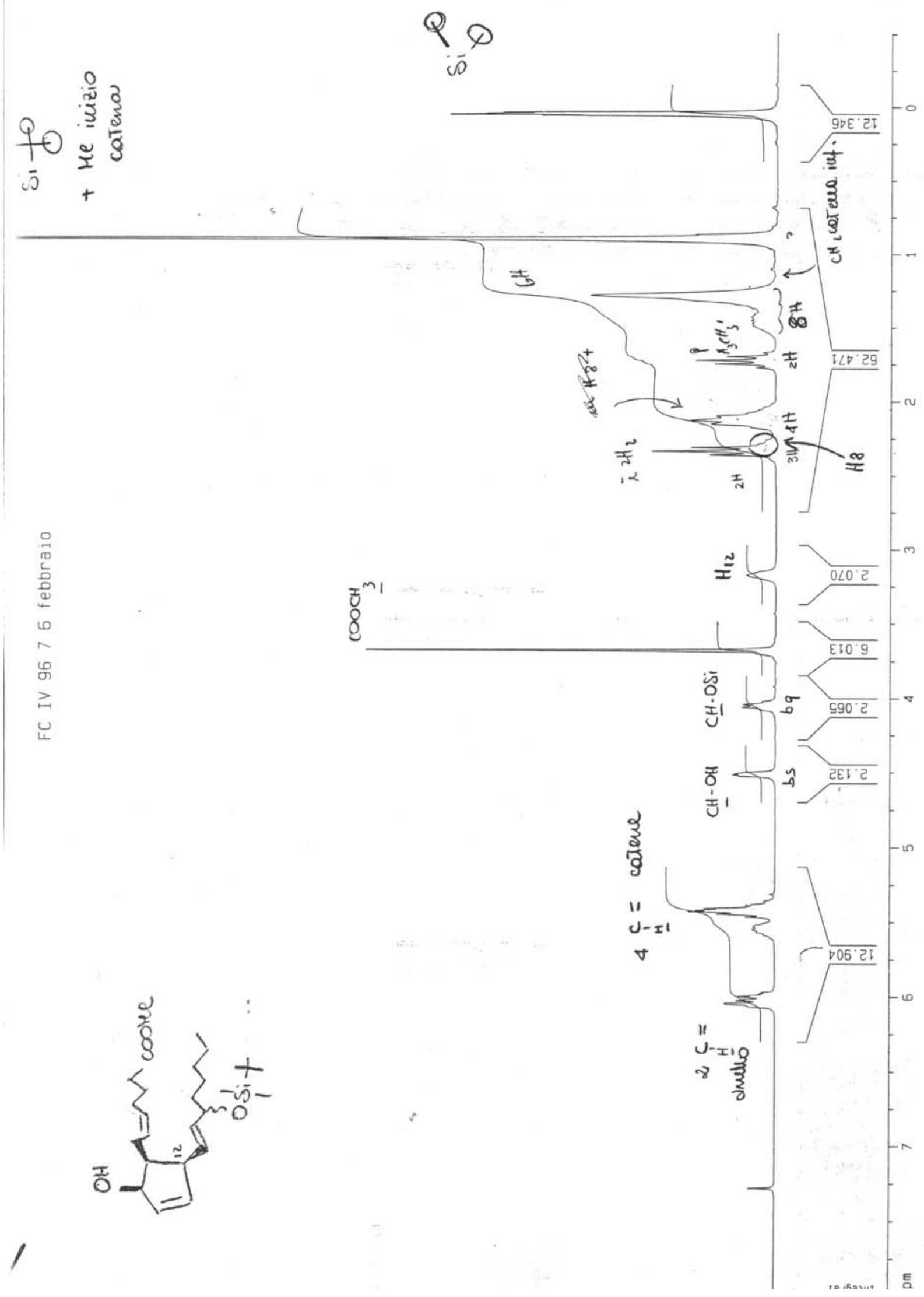




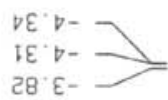

$28,8-1$

EQ 81

$\checkmark 0^{\circ} \varepsilon 己$

$0 D^{\circ} \mathrm{DC}$

$\left.\nabla \nabla_{0} \nabla\right]$

मट. द्व

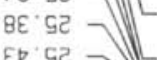

es.

6! $\angle 2$

$O{ }^{\circ}$ टE

BE' $\mathrm{CE}$

$\triangle 6^{\circ} \mathrm{EE}$.

SB BE

$88.8 E$

$O E^{\prime} \angle D$

$00.05 \longrightarrow$

टC.05

88 . Is

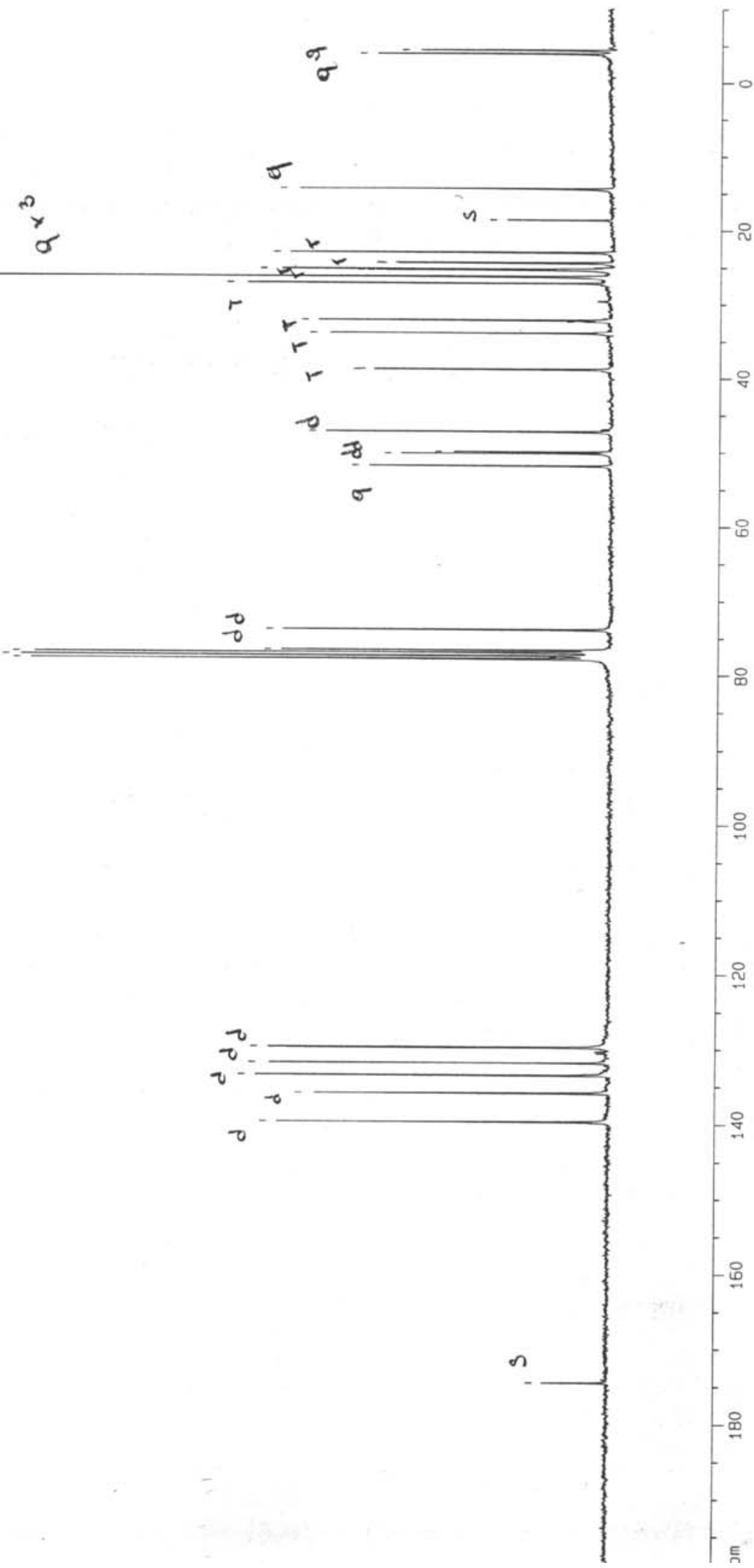

08621

58.621-

$68^{\circ}$ IEI

GS $E E I-$

EG GEI-

GL'GEI-

IS $\nabla<1$

wdd 
$\varepsilon \cdot D-\longrightarrow$

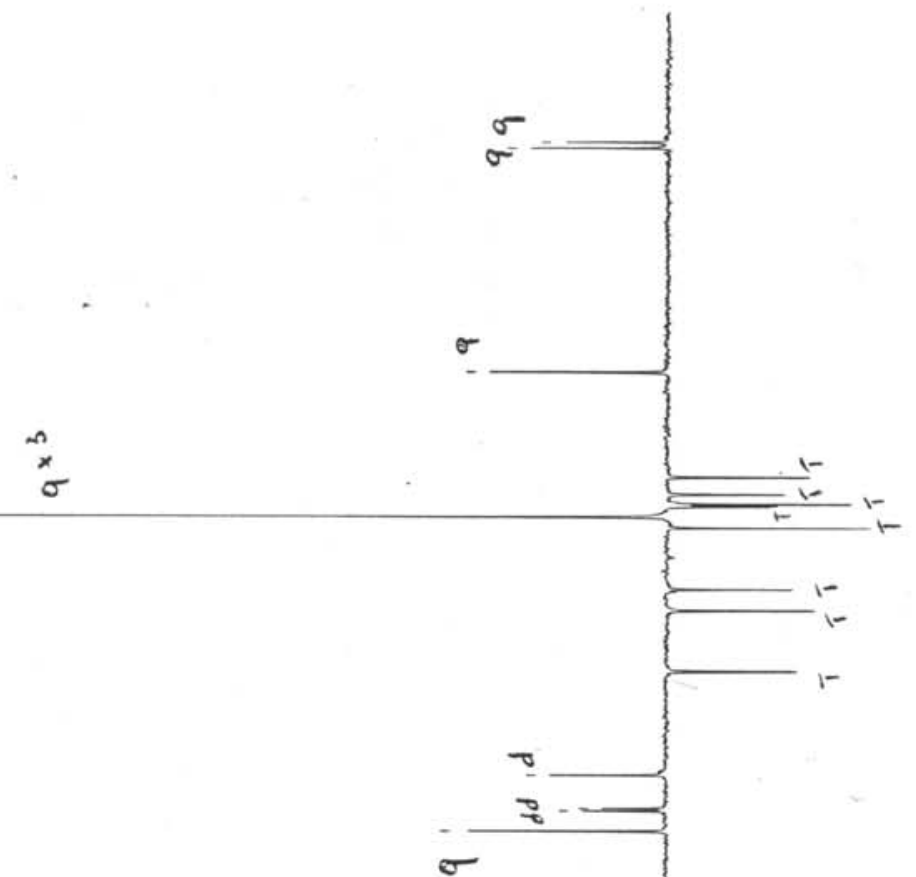

ஜ

구 $\left.\begin{array}{ll}0 . E C \\ \nabla . \Delta L\end{array}\right]$

$\left.\begin{array}{ll}0 . \Delta C \\ 0.12\end{array}\right]$

$\left.\begin{array}{ll}8.95 \\ 0.05\end{array}\right]$

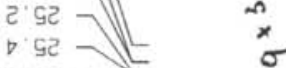

$\checkmark \mathrm{SC}$

0.
S.

S. $2 E$

S. CE

B. $B E$

6. $B E$

$E \angle D=$

0.05

2.05

$6.15-$

$\sigma$

$6 . E L$
$\angle 9 L$

$8 \cdot 521-$

$8.651 \longrightarrow$

6. IE!

9 EEI-

6. GEI -

B. $6 E$ I

wdd

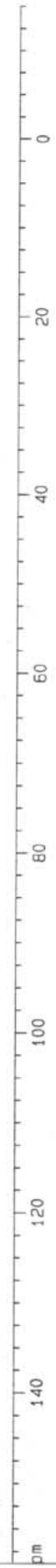




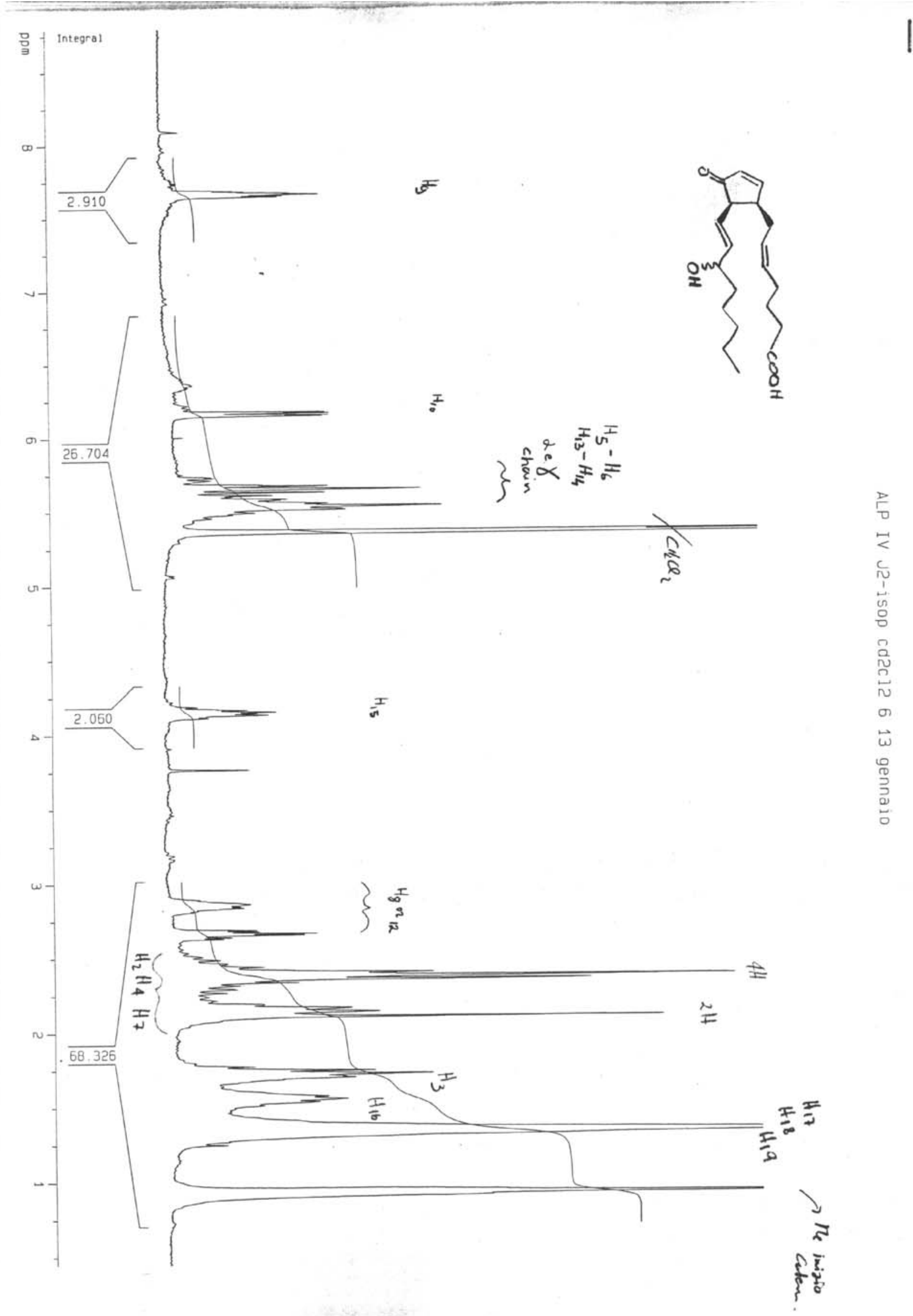




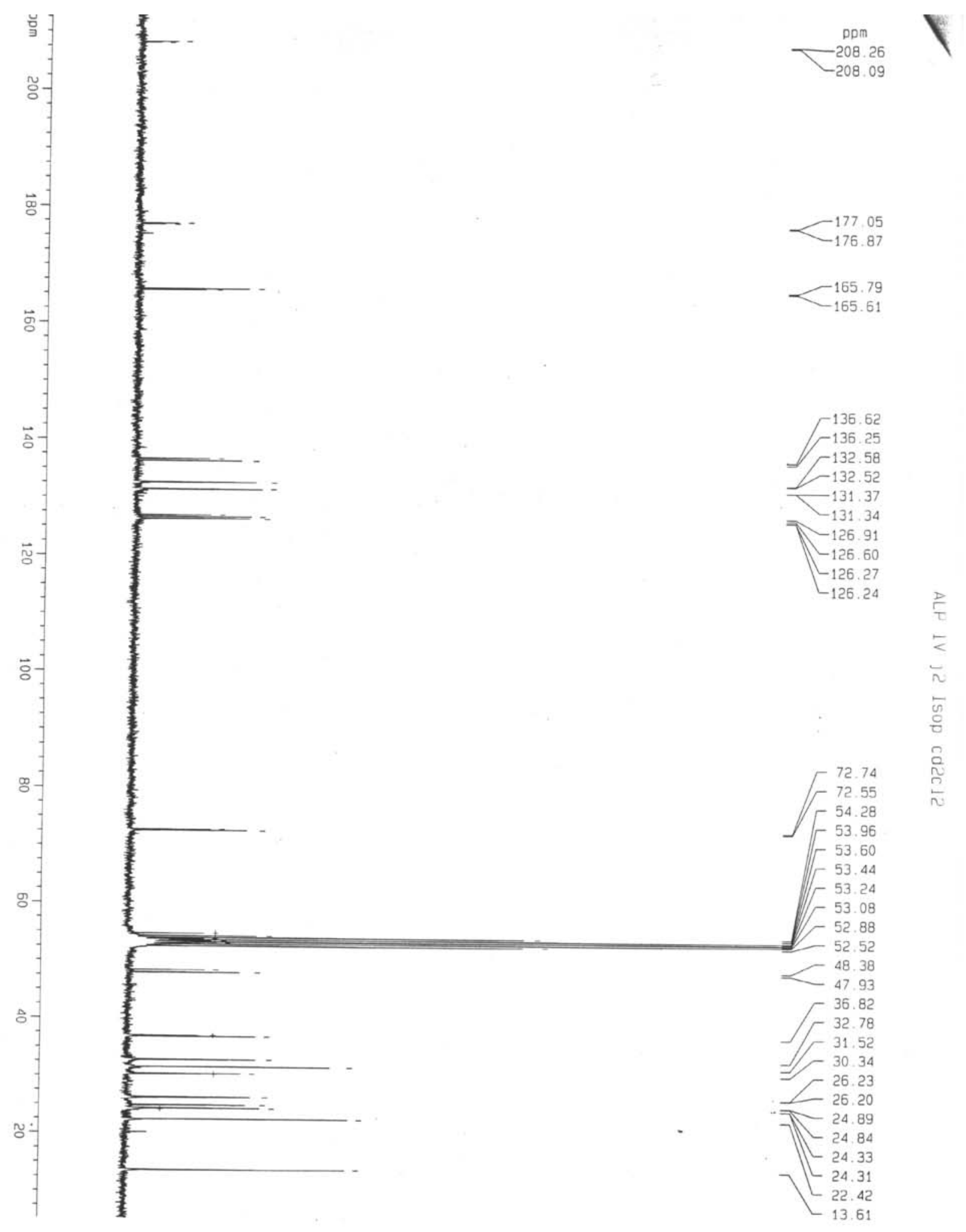

